\title{
Effects of panretinal photocoagulation on macular vascular density and Foveal avascular zone area in patients with severe non-proliferative diabetic retinopathy by optical coherence tomography angiography
}

Hongjing Zhu

The First Affiliated Hospital of Nanjing Medical University

Weiwei Zhang

The First Affiliated Hospital of Nanjing Medical University

Qinghuai Liu ( $\square$ liuqh@njmu.edu.cn )

The First Affiliated Hospital of Nanjing Medical University

\section{Research Article}

Keywords: optical coherence tomography angiography, diabetic retinopathy, Panretinal photocoagulation, vascular density

Posted Date: December 9th, 2020

DOl: https://doi.org/10.21203/rs.3.rs-118201/v1

License: (9) This work is licensed under a Creative Commons Attribution 4.0 International License. Read Full License 


\section{Abstract}

Aim: To describe the changes of optical coherence tomography angiography (OCTA) characteristics in severe non-proliferative Diabetic Retinopathy(S-NPDR) eyes. And study the effect of Panretinal photocoagulation(PRP) on these characteristics.

Methods: This is a prospective study including 31 eyes from 18 consecutive patients with S-NPDR and 31 eyes of healthy subjects. We measured macular vascular density (VD) and foveal avascular zone(FAZ) area by an OCTA device. All patients with S-NPDR underwent PRP treatment. In S-NPDR eyes, the VD and foveal avascular zone(FAZ) area were assessed at 1 week before PRP, 1 month, 3 months and 6 months following PRP.

Results: Compared with the normal control group ,in superficial retinal capillary plexus (SCP), macular VD decreased in S-NPDR group expect foveal VD. In foveal,$p=0.7$; In parafoveal and perifoveal, $p \otimes 0.001$. In deep retinal capillary plexus (DCP), macular VD was also lower in S-NPDR group. In foveal, $p=0.01$.In

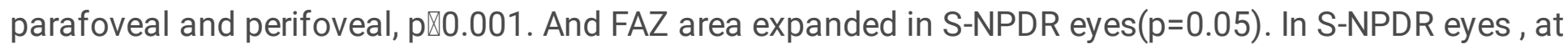
3 months after PRP, foveal VD in DCP increased significantly $(p=0.04)$. At 6 months after PRP, foveal VD increased both in SCP and DCP ( $P=0.01, P=0.008$,respectively). At 6 months after PRP,FAZ area decreased $(p=0.04)$.

Conclusions : Patients with S-NPDR have retinal microcirculation disorder, and PRP can partially improve the macular microcirculation. VD and FAZ can be used as sensitive indicators for follow-up observation of diabetic retinopathy.

\section{Introduction}

Diabetic retinopathy(DR) is a common microvascular complication of diabetes, and remains the leading cause of blindness in working-aged people. Clinically, DR is divided into non proliferative DR(NPDR) and proliferative $\mathrm{DR}(\mathrm{PDR})$, which is marked by the appearance of retinal neovascularization(1). Panretinal photocoagulation (PRP) has been standard treatment for PDR since the advent of ophthalmic lasers. The aim of PRP is to cause regression of retinal neovascularisation in retina ,reduce the risk of vitreous hemorrhage, tractional retinal detachment and thereby prevent the progression of visual loss(2).Although PRP has been widely used in clinic, its mechanism is not very clear. The main hypothesis of the mechanism for PRP is that the PRP damages some of the highly metabolically photoreceptors, which consume lots of oxygen(3).This process may improve the oxygen supply to the inner retina, and reach a new balance between oxygen supply and demand, to reduce the stimulus for neovascularization. Besides ,the decreased area of retinal tissue leads to improved oxygenation and a reduction in the levels of vascular endothelial growth factor(VEGF). A reduction in levels of VEGF may be important in reducing the risk of harmful new vessels forming.

Previous studies have investigated the change of ocular circulation after PRP and reported PRP reduces retinal blood flow (4). However, these studies concentrated on blood flow of whole retina rather than 
macula, which is more important and particular for the maintenance of visual function. According to our expectation, when the laser was placed on the peripheral parts of retina, it would cause thermal damage to outer retinal tissues, and blood will be distributed from periphery to macula region. It means although blood flow decreases in whole retina, the blood flow to macula may increase after PRP. FAZ is a capillaryfree area surrounded by separation of inner retinal layers. It is highly sensitive to ischemic events and can be an indicator of several pathologic processes. In our expectation, FAZ area may remain unchanged or decreased following effective PRP.

Optical coherence tomography angiography (OCTA) is a new, noninvasive imaging technique that generates real-time data on vascular structures of eye without intravenous injection of fluorescent dyes. By OCTA, we can get information of individual layers and different parts of retina. Besides, OCTA becomes a new tool for follow-up observation of changes in FAZ(5). Using OCTA, we evaluated the changes of macular VD and FAZ area between healthy eyes and S-NPDR eyes. And then we studied the long-term effects of PRP on macular VD and FAZ areas of S-NPDR patients after PRP.

\section{Materials And Methods}

\section{Design and population}

The study followed the tenets of the Declaration of Helsinki, and was approved by the Ethics Committee of First Affiliated Hospital of Nanjing Medical University (2020-SR-269). This study prospectively analyzed patients with S-NPDR who had recently received PRP in the First Affiliated Hospital of Nanjing Medical University. The S-NPDR patients who were ready to receive PRP, and age-matched healthy subjects were included in the study. All participants or guardians were informed consent. And all participants were not involved in the design,or conduct, or reporting, or dissemination plans of our research.

The inclusion criteria of healthy group: (1).aged 45-65; (2). - $2.0 \mathrm{D} \leq$ spherical equivalent (SE) $\leq 2.0 \mathrm{D}$; (3). intraocular pressure (IOP) $\leq 21 \mathrm{mmhg}$ and without eye diseases (such as glaucoma and fundus disease); (4). without ophthalmic surgery and trauma history; (5).without diabetes. Inclusion criteria of S-NPDR subjects:(1) diagnosed as S-NPDR by two experienced ophthalmologists respectively; (2) underwent PRP and were followed at least 6 months; (3) without ocular history which affects retina and choroid such as vascular occlusion, retinal neovascularization, inflammatory disease, glaucoma, or trauma;(4) without previous vitreous surgery, anti-VEGF or photocoagulation before this study and without any additional therapy including macular photocoagulation or anti-VEGF during the study ; (5) without clinically significant macular edema before PRP; (6)without corneal opacity, severe cataract, that could influence image quality. Fluorescein angiography (FA) and fundus photo were performed before PRP. Slit-lamp biomicroscopy, Goldmann applanation tonometry and OCTA were performed at every follow-up.

\section{Panretinal photocoagulation treatment}

According to the recommendations of the Early Treatment Diabetic Retinopathy Study Group, PRP was applied in 4 peripheral quadrants in 2 to 4 sessions by an experienced ophthalmologist who was unaware 
of the study design. All photocoagulation sessions were performed with an interval of 1 week. A total of 1200 to 2000 spots were applied per eye with the spot size of $200-500 \mu \mathrm{m}$ and pulse duration of 0.2 seconds.

\section{Optical coherence tomography angiography}

Optical coherence tomography angiography scans were obtained using AngioVue (version 2017.1.0.151); Optovue, Fremont, CA, USA) with the angio retina mode $(6 \times 6 \mathrm{~mm})$. Automatic segmentation was performed by the viewing software to generate en face projection images of the superficial retinal capillary plexus (SCP), deep retinal capillary plexus (DCP). The SCP is segmented from inner internal limiting membrane to $9 \mu \mathrm{m}$ above lower boundary of inner plexiform layer. The DCP is segmented from $9 \mu \mathrm{m}$ above inner plexiform layer to $9 \mu \mathrm{m}$ below lower boundary of outer plexiform layer(Fig. 1). To reduce the effect of artifacts and segmentation errors, we excluded images with severe artifacts $(\leq 6 / 10)$ and performed manual correction of the segmentation of retina in every image by a well-trained ophthalmologist. Then, images were checked by another two ophthalmologists, only when both of them agreed the segmentation, can an image be used in analysis.Angiography information calculated by embedded software in the machine comprises the area of the foveal avascular zone (FAZ), and the VD of SCP, DCP. VD was defined as the percentage area occupied by vessels. All OCTA examinations were performed by the well-trained studier.

\section{Statistical analysis}

Statistical analysis was performed using an SPSS software package (SPSS 22.0). Independent samples t-test was used to compare the change of VD and FAZ area. $P<0.05$ is considered as statistically significant.

\section{Results}

\section{Patient demographics}

A total of 80 eyes were recruited in our study, and 18 of these eyes were excluded because of poor image quality and wrong segmentation of retina. Demographic and anatomic characteristics of patients included in this study are summarized in Table 1. In S-NPDR eyes which received PRP, mean log MAR BCVA at baseline $(0.4 \pm 0.2)$ showed no significant change $(p=0.6)$ with the last visit after $\operatorname{PRP}(0.4 \pm 0.1)$. Mean $\mathrm{HbA} 1 \mathrm{c}$ at baseline was $5.6 \pm 1.2(\%)$, which also had no significant change $(p=0.5)$ to $5.4 \pm 1.4(\%)$ at the last visit. 
Table 1

Clinical characteristics of patients at baseline

\begin{tabular}{|lll|}
\hline Characteristics & S-NPDR eyes & Normal eyes \\
\hline Gender,no.(\%) Men / Women & $15(48 \%) / 16(52 \%)$ & $17(51 \%) / 14(49 \%)$ \\
\hline Mean patient age (IQR),years & $58 \pm 12.2(50-66)$ & $54 \pm 9.17(46-58)$ \\
\hline Mean log MAR BCVA (IQR) & $0.4 \pm 0.2(0.2-0.5)$ & $0 \pm 0.03(0-0)$ \\
\hline Duration of diabetes (IQR),years & $14 \pm 4.8(10-17)$ & - \\
\hline Type of DM,no.(\%) Type 1 Type2 & $4(12 \%) 27(88 \%)$ & - \\
\hline HbA1c (\%) (IQR) & $5.6 \pm 1.2(4.5-7.0)$ & - \\
\hline Lens status ,no(\%) Phakic Pseudophakic & $29(94 \%) 2(6 \%)$ & $31(100 \%) 0(0 \%)$ \\
\hline hypertension,no.(\%) & $6(19 \%)$ & $5(16 \%)$ \\
\hline $\begin{array}{l}\text { IQR:interquartile range; log MAR: logarithm of the minimum angle of resolution; BCVA:Best-corrected } \\
\text { visual acuity }\end{array}$ & \\
\hline
\end{tabular}

\section{Macular VD between normal eyes and S-NPDR eyes before PRP}

Table 2 shows the difference between healthy eyes and S-NPDR eyes before PRP. In SCP, macular VD was significantly lower in S-NPDR group than that in normal subjects expect fovea VD. $(p<0.001, p=0.7)$. In DCP, macular VD was significantly lower in S-NPDR group than that in normal eyes. In fovea, $p=0.01$, and in parafovea and perifovea, $p<0.001$.

Table 2. The difference of VD and FAZ area between healthy eyes and S-NPDR eyes before PRP 


\begin{tabular}{|c|c|c|c|c|}
\hline variables & & healthy control & S-NPDR before PRP & P value \\
\hline \multirow[t]{4}{*}{ SCP vascular density (\%) } & Whole & 51.37 & 45.26 & $<0.001 * *$ \\
\hline & fovea & 17.6 & 16.99 & 0.7 \\
\hline & parafovea & 53.6 & 44.6 & $<0.001 * *$ \\
\hline & perifovea & 52.3 & 46.11 & $<0.001 * *$ \\
\hline \multirow[t]{4}{*}{ DCP vascular density (\%) } & Whole & 53.75 & 44.19 & $<0.001$ ** \\
\hline & fovea & 32.45 & 27.24 & $0.01 * \star$ \\
\hline & parafovea & 56.78 & 48.74 & $<0.001 * *$ \\
\hline & perifovea & 54.5 & 45.01 & $<0.001 * *$ \\
\hline $\mathrm{FAZ}$ area $\left(\mathrm{mm}^{2}\right)$ & & 0.309 & 0.364 & 0.04 * \\
\hline
\end{tabular}

Table 3 shows the changes of VD in S-NPDR eye after PRP. Three months after PRP, the VD of DCP fovea increased first $(p=0.04)$. Six months after PRP, the foveal VD of SCP and DCP increased significantly $(p=$ $0.01, p=0.008$,respectively). There was no significant change in blood vessel density in other parts of the macula. Figure 2 illustrates the trend of VD in normal eyes, S-NPDR eyes before and after PRP.

Table 3. Changes of VD and FAZ area in S-NPDR eyes following PRP 


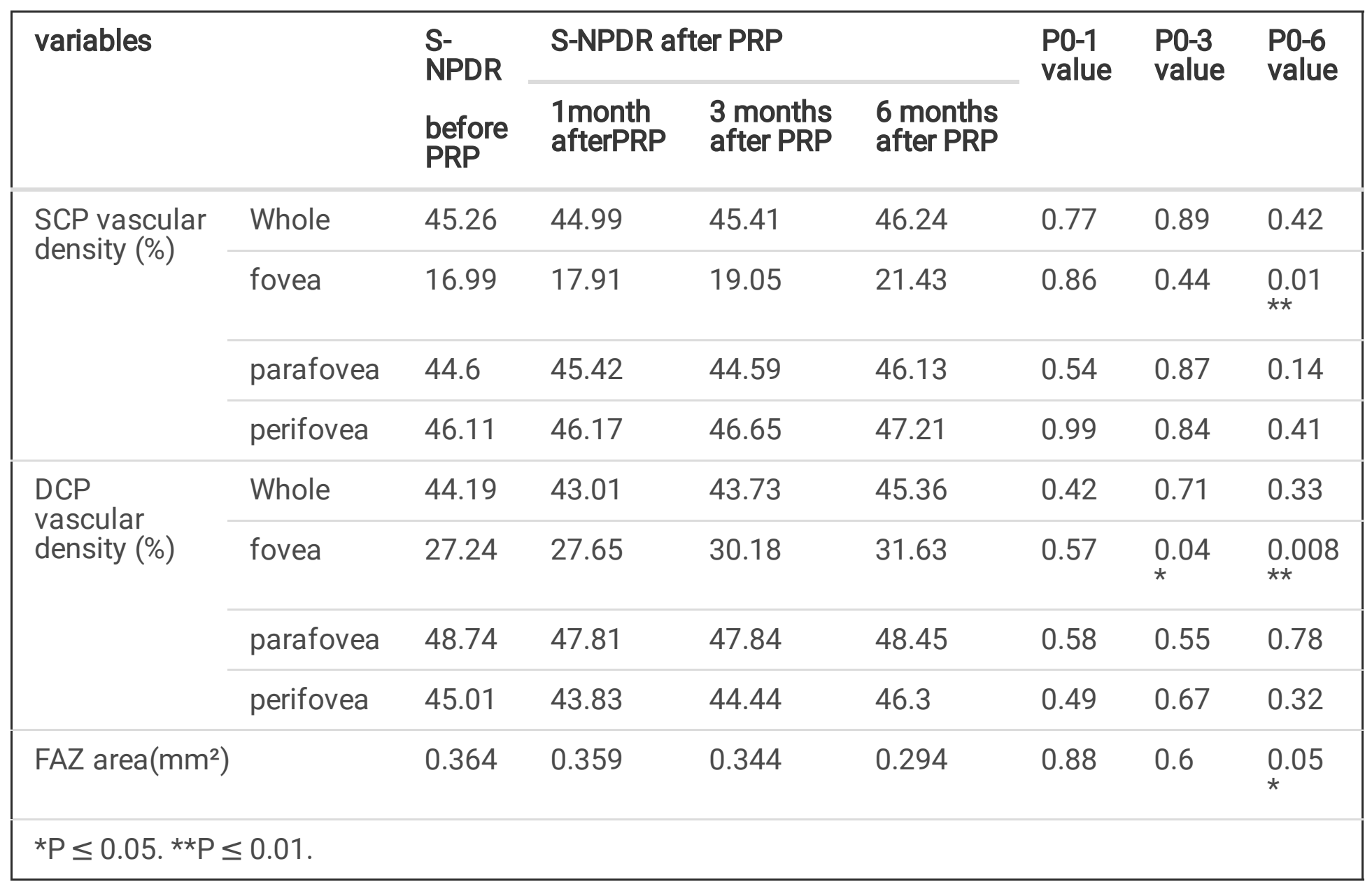

\section{FAZ area}

Tables 2 and 3 show that, compared with healthy eyes, the FAZ area of S-NPDR eyes increased $(p=0.05)$. In S-NPDR eyes receiving PRP, the area of FAZ decreased significantly 6 months after surgery $(p=0.04)$.

\section{Discussion}

In this prospective study, we found that S-NPDR eyes showed a significant decrease macular VD except fovea VD of SCP before PRP. Besides, FAZ area increased significantly. In S-NPDR eyes treated with PRP, VD only changed in fovea. Three months after PRP, the VD in fovea of DCP first increased significantly. Six months after PRP, the VD in fovea of SCP and DCP both increased. And FAZ area had a significant decrease at 6 months following PRP.

Consistent with previous studies $(6,7)$, our results showed whole en face VD decreased in SCP and $\operatorname{DCP}(p<0.001)$ in S-NPDR eyes. It should be noted, however, this change was less remarkable in fovea. In parafovea and perifovea, VD decreased both in SCP and DCP $(p<0.001)$. In fovea, VD of SCP showed a non-significant decrease $(17.6 \%-16.99 \%, p=0.7)$ and VD of DCP decreased from $32.45-27.49 \%(P=$ 0.01). Perhaps it was because DR affects the peripheral retina first, and fovea is damaged in the last stage. In addition, fovea is a region which overlies photoreceptors and has a high acuity vision. 
(8)Another possibility is that relatively preserved fovea VD helps to maintain visual acuity and it may be a self-protective mechanism of retina. Of course, there is another possibility that fovea has fewer capillary than parafovea and perifovea, so the change of VD is not obvious. Further studies are needed to clarify the mechanism. Besides, we had an unexpected finding that fovea VD of SCP did not decreased significantly in S-NPDR eyes $(p=0.7)$. But fovea VD of DCP decreased significantly $(P=0.01)$. So far OCTA examinations have been performed on various layers of the retina of DR patients. In the early stage of DR, SCP showed high flow and DCP presented a steep decline of blood flow with increasing DR severity $(6,9,10)$. Compared with SCP, blood flow of DCP decreased more steeply with the aggravation of DR. These findings coincide with our result of fovea VD decreased only in DCP. One possible explanation is the self-regulation of SCP. With the progress of DR, SCP retains a certain degree of self-regulation function compared with DCP. Another possible explanation is that the dilated capillaries of SCP, which have increased blood flow and decreased vascular resistance, may contribute a steal phenomenon at DCP.(11)

In S-NPDR eyes which received PRP, fovea VD increased. At 3 months following PRP, fovea VD in DCP increased first $(p=0.03)$. At 6 months following PRP, fovea VD increased both in SCP and DCP $(p=0.01, p$ $=0.008$, respectively). The changes of VD in S-NPDR eyes which received PRP were consistent to our expectation. However, the mechanism of this observation is not fully clarified. Therefore ,we proposed several hypotheses. The first assumption we put forward is that PRP may redistribute the circulation of ocular. It is generally accepted that laser energy is mainly absorbed by retinal pigment epithelium (RPE), destroys RPE and photoreceptor cells, and injures surrounding retina and choriocapillaris. Both in retina and choroid, capillary dropout in the areas of laser scars(12). There are some studies have suggested that choroidal vessels in the untreated macular area increased after retinal peripheral photocoagulation $(13,14)$. For example, Takahashi reported PRP may increase choroidal blood flow in fovea in eyes with severe diabetic retinopathy (14). So, it is also possible that fovea VD in retina increased after PRP. Histological studies should be performed to prove this assumption. The second assumption is the recanalisation occurred in fovea vascular after PRP. As we all know, DR is a form of microangiopathy which accompanied by obstruction of capillaries. (15) Several ophthalmologists have demonstrated the occurrence of capillary revascularization(16, 17). Rema Mohan and Evam Kohner reported a case which demonstrated the occurrence of capillary revascularization in a previously avascular area of the retina in a patient with advanced diabetic eye disease after extensive photocoagulation. Meanwhile, the vessels are in the plane of the retina and do not have the appearance of new vessels(16). By optics scanning light ophthalmoscopy, Chui, T.Y. observed capillary recanalized and remained perfused in fovea (17). These examples illustrated macular VD is reversible and it is possible that there are partially reversible components in DR. Thus, we hypothesized that PRP may promote the recanalization of fovea microvascular. More studies are needed to verify these assumptions.

Interestingly, both fovea VD of SCP and DCP increased, but the enhancement of DCP was more notable and earlier. In our investigation between S-NPDR eyes and normal eyes, S-NPDR eyes showed a significant decrease in fovea VD of $\operatorname{DCP}(p=0.01)$, while fovea VD of SCP had no significant change $(p=$ 0.7). Besides, as we have mentioned, many previous studies have suggested microvascular changes 
occur at DCP earlier than SCP in DR $(6,9,10)$. These above two points may help explaining the phenomenon that DCP is more susceptible to PRP. Previous studies together with our observations suggest a role of DCP in evaluating therapeutic effect and monitoring DR progression.

Furthermore, we found FAZ area in S-NPDR eyes increased $(p=0.05)$ before PRP. At 6 months following PRP, FAZ area decreased $(p=0.04)$. In $D R$, an increase in FAZ area caused by capillary alteration has already been shown and it is in line with our finding(18). The FAZ is a specialized region of the human retina which is capillary-free and approximates the region of highest cone photoreceptor density and oxygen consumption. (19) It is well known that diabetes induces microvascular diseases including not only retinopathy but also choroidopathy, and the disrupted choroidal circulation also has clinical relevance and contributes to the pathogenesis. Cells in FAZ are principally nourished by the choroid and during physiologic conditions the choroidal circulation is able to meet the metabolic demands of the $F A Z(20)$. And the severity of DR is associated with enlarged FAZ area(21). Our finding that FAZ area decreased after PRP suggests PRP could reverse the progression of DR. Consequently, FAZ area may be a marker for monitoring the therapeutic effect in DR.

Before our study, Fawzi et.al studied 10 DR eyes and found VD decreased only in middle capillary plexuses which is located between SCP and DCP after PRP(22). But they only studied whole en face VD of retina and ignore different change in different region of macula and FAZ area.

Of course, there are several limitations in this study. Firstly, it is impossible for OCTA machine to avoid all of artifacts and segmentation errors of retina, particularly in eyes with poor vision. To reduce the effect of artifacts and segmentation errors, manual adjustments were performed to segment retina and edge FAZ in every image. Secondly, during our follow-up period, five eyes appeared cystoid macular edema. Some previous studies reported a decrease of macular VD in patients with macular edema. $(23,24)$ So we supposed that macular edema will not make VD decreased. In addition, anti-VEGF therapy has been widely applied in recent years and has achieved short-term returns. However, anti-VEGF therapy needs high costs, regular follow-up and it is still to be studied whether it will relapse after anti-VEGF withdrawal. If we can combine anti-VEGF and PRP, the macular edema after PRP may be inhibited. We are also carrying out relevant research. Last but not least, the patient numbers in our study was still limited. Future studies with larger samples are needed to verify our results.

In summary, by compared with healthy eyes, we found macular VD decreased in S-NPDR eyes, and the decrease of fovea VD is not as significant as parafovea and perifovea. After PRP, only fovea VD increase, especially in DCP. It suggests us fovea VD could be a possible predictor of the effectiveness of PRP. In addition, FAZ area decreased at 6 months after PRP. It suggested FAZ area may also help in the assessment of treatment efficacy. Our study provided a new angle to the study of mechanisms of PRP and further work is needed to understand the effect of PRP better. 


\section{Declarations}

\section{Ethics approval and consent to participate}

The study followed the tenets of the Declaration of Helsinki, and was approved by the Ethics Committee of First Affiliated Hospital of Nanjing Medical University (2020-SR-269). All participants or guardians were informed consent.

\section{Consent for publication}

Not applicable.

\section{Availability of data and materials}

The datasets used and/or analysed during the current study available from the corresponding author on reasonable request.

\section{Competing interests}

The authors declare that they have no competing interests.

\section{Funding}

This study was supported by National key research and development program (2017YFA0104101) and Six Talent Peaks Project in Jiangsu Province of China(WSN-002).

\section{Authors' contributions}

W.Z. and Q.L.conceived and designed the study. H.Z. conducted the follow-up of patients. H.Z.interpreted data and drafteded manuscript. W.Z. and Q.L. revised the manuscript. The datasets used and/or analysed during the current study are available from the corresponding author on reasonable request.

\section{Ackonwledgements}

We would like to thank all of the paticipants involved in this study.

\section{References}

1. Lee R, Wong TY, Sabanayagam C. Epidemiology of diabetic retinopathy, diabetic macular edema and related vision loss. Eye and Vision. 2015;2(1).

2. Ockrim Z, Yorston D. Managing diabetic retinopathy. BMJ. 2010;341:c5400.

3. Birol G, Wang S, Budzynski E, Wangsa-Wirawan ND, Linsenmeier RA. Oxygen distribution and consumption in the macaque retina. Am J Physiol Heart Circ Physiol. 2007;293(3):H1696-704. 
4. Feke GT, Green GJ, Goger DG, McMeel JW. Laser Doppler Measurements of the Effect of Panretinal Photocoagulation on Retinal Blood Flow. Ophthalmology. 1982;89(7):757-62.

5. Kashani AH, Chen CL, Gahm JK, Zheng F, Richter GM, Rosenfeld PJ, et al. Optical coherence tomography angiography: A comprehensive review of current methods and clinical applications. Prog Retin Eye Res. 2017;60:66-100.

6. Simonett JM, Scarinci F, Picconi F, Giorno P, De Geronimo D, Di Renzo A, et al. Early microvascular retinal changes in optical coherence tomography angiography in patients with type 1 diabetes mellitus. Acta Ophthalmol. 2017;95(8):e751-e5.

7. Sun Z, Tang F, Wong R, Lok J, Szeto SKH, Chan JCK, et al. OCT Angiography Metrics Predict Progression of Diabetic Retinopathy and Development of Diabetic Macular Edema. Ophthalmology. 2019;126(12):1675-84.

8. Bringmann A, Syrbe S, Görner K, Kacza J, Francke M, Wiedemann P, et al. The primate fovea: Structure, function and development. Progress in Retinal and Eye Research. 2018;66:49-84.

9. Hwang TS, Zhang M, Bhavsar K, Zhang X, Campbell JP, Lin P, et al. Visualization of 3 Distinct Retinal Plexuses by Projection-Resolved Optical Coherence Tomography Angiography in Diabetic Retinopathy. JAMA Ophthalmol. 2016;134(12):1411-9.

10. Nesper PL, Roberts PK, Onishi AC, Chai H, Liu L, Jampol LM, et al. Quantifying Microvascular Abnormalities With Increasing Severity of Diabetic Retinopathy Using Optical Coherence Tomography Angiography. Investigative Opthalmology \& Visual Science. 2017;58(6).

11. Onishi AC, Nesper PL, Roberts PK, Moharram GA, Chai H, Liu L, et al. Importance of Considering the Middle Capillary Plexus on OCT Angiography in Diabetic Retinopathy. Investigative Opthalmology \& Visual Science. 2018;59(5).

12. Karst SG, Beiglboeck H, Scharinger R, Meyer EL, Mitsch C, Scholda C, et al. Retinal and Choroidal Perfusion Status in the Area of Laser Scars Assessed With Swept-Source Optical Coherence Tomography Angiography. Invest Ophthalmol Vis Sci. 2019;60(14):4865-71.

13. Flower RW, Fryczkowski AW, McLeod DS. Variability in choriocapillaris blood flow distribution. Invest Ophthalmol Vis Sci. 1995;36(7):1247-58.

14. Takahashi A, Nagaoka T, Sato E, Yoshida A. Effect of panretinal photocoagulation on choroidal circulation in the foveal region in patients with severe diabetic retinopathy. $\mathrm{Br} \mathrm{J}$ Ophthalmol. 2008;92(10):1369-73.

15. Muraoka K, Shimizu K. Intraretinal Neovascularization in Diabetic Retinopathy. Ophthalmology. 1984;91(12):1440-6.

16. Mohan R, Kohner EM. Retinal revascularisation in diabetic retinopathy. Br J Ophthalmol. 1986 Feb;70(2):114-7.. (Mohan R, Kohner EM. Retinal revascularisation in diabetic retinopathy. $\mathrm{Br} \mathrm{J}$ Ophthalmol. 1986 Feb;70(2):114-7. doi: 10.1136/bjo.70.2.114.).

17. Chui TY, Pinhas A, Gan A, Razeen M, Shah N, Cheang E, et al. Longitudinal imaging of microvascular remodelling in proliferative diabetic retinopathy using adaptive optics scanning light ophthalmoscopy. Ophthalmic Physiol Opt. 2016;36(3):290-302. 
18. Freiberg FJ, Pfau M, Wons J, Wirth MA, Becker MD, Michels S. Optical coherence tomography angiography of the foveal avascular zone in diabetic retinopathy. Graefes Arch Clin Exp Ophthalmol. 2016;254(6):1051-8.

19. Yu DY, Cringle SJ, Su EN. Intraretinal oxygen distribution in the monkey retina and the response to systemic hyperoxia. Invest Ophthalmol Vis Sci. 2005;46(12):4728-33.

20. Balaratnasingam C, Inoue M, Ahn S, McCann J, Dhrami-Gavazi E, Yannuzzi LA, et al. Visual Acuity Is Correlated with the Area of the Foveal Avascular Zone in Diabetic Retinopathy and Retinal Vein Occlusion. Ophthalmology. 2016;123(11):2352-67.

21. Tang FY, Ng DS, Lam A, et al. Determinants of Quantitative Optical Coherence Tomography Angiography Metrics in Patients with Diabetes [published correction appears in Sci Rep. 2018 May 4;8(1):7314]. Sci Rep. 2017;7(1):2575. Published 2017 May 31. doi:10.1038/s41598-017-02767-0.

22. Fawzi AA, Fayed AE, Linsenmeier RA, Gao J, Yu F. Improved Macular Capillary Flow on Optical Coherence Tomography Angiography After Panretinal Photocoagulation for Proliferative Diabetic Retinopathy. Am J Ophthalmol. 2019;206:217-27.

23. Toto L, D’Aloisio R, Di Nicola M, Di Martino G, Di Staso S, Ciancaglini M, et al. Qualitative and Quantitative Assessment of Vascular Changes in Diabetic Macular Edema after Dexamethasone Implant Using Optical Coherence Tomography Angiography. International Journal of Molecular Sciences. 2017;18(6).

24. Serra R, Sellam A, Coscas F, Bruyere E, Sieiro A, Coscas GJ, et al. Evaluation of pseudophakic cystoid macular edema using optical coherence tomography angiography. Eur $\mathrm{J}$ Ophthalmol. 2018;28(2):234-40.

\section{Figures}


SCP
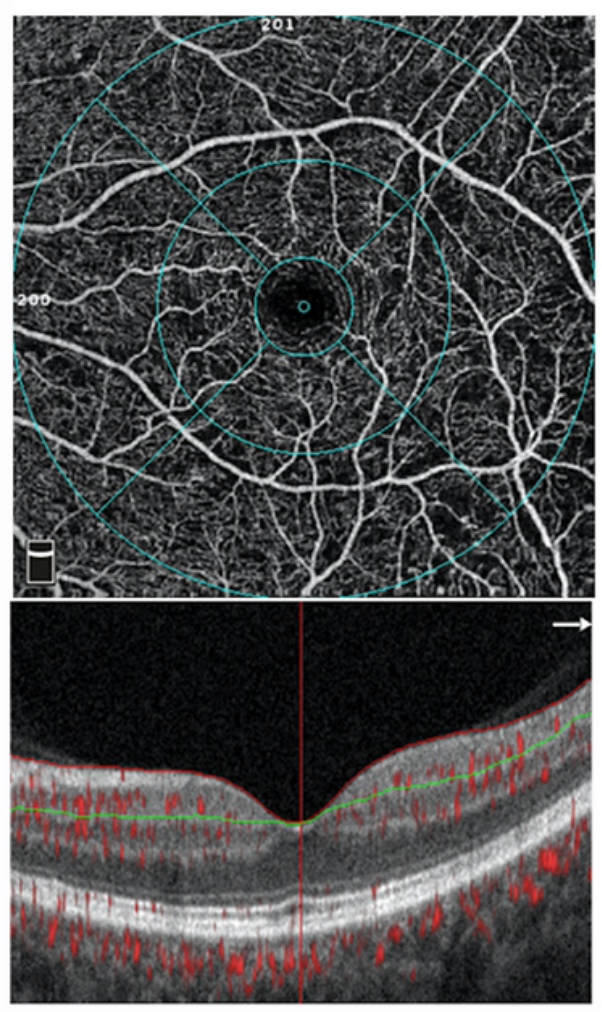

DCP
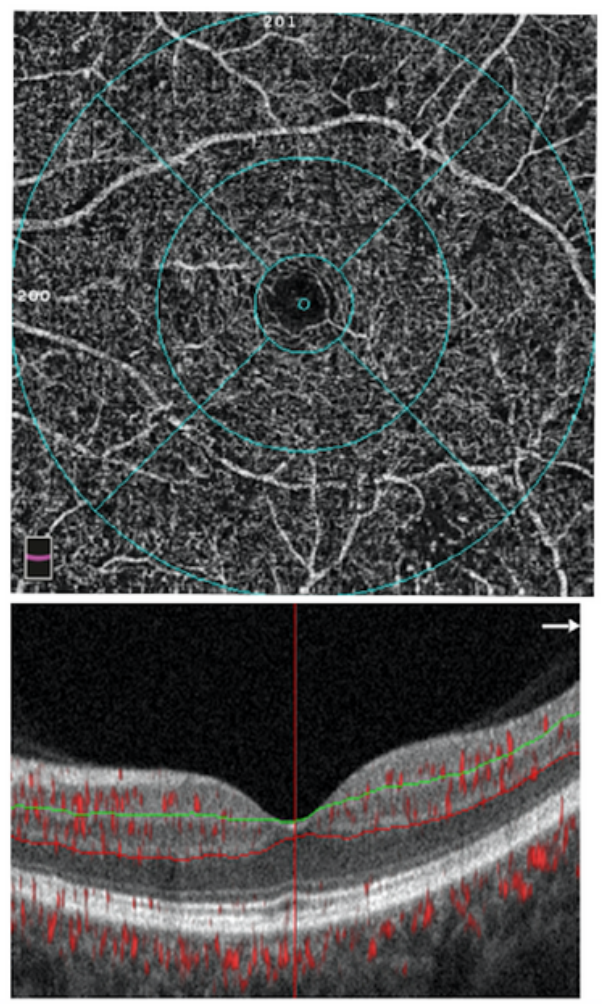

FAZ
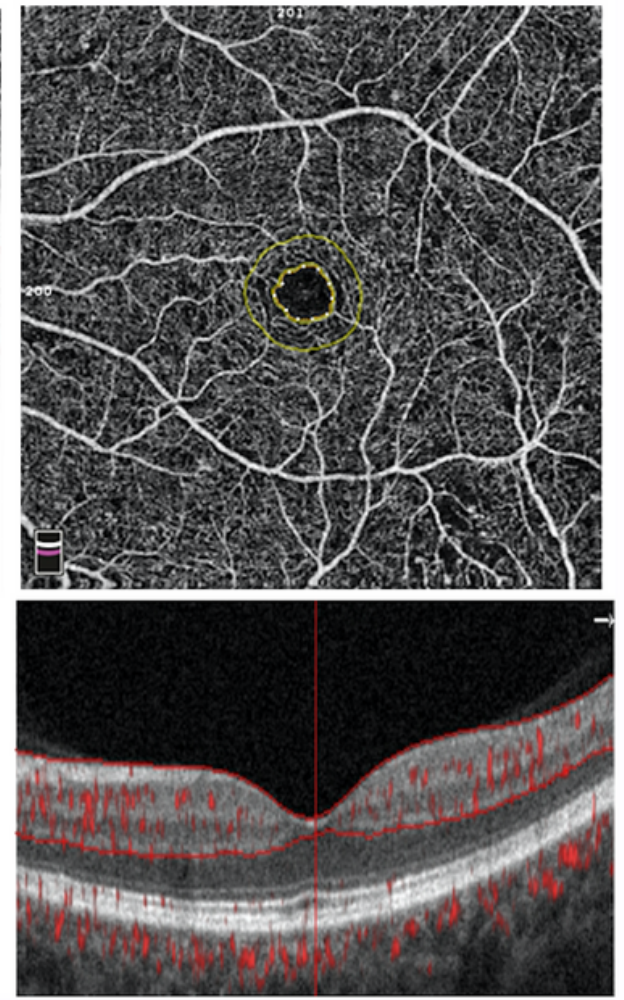

\section{Figure 1}

The segmentation of retinaEn face OCTA image of SCP(left),DCP(middle) and FAZ (right) of a S-NPDR eye. Segmentation boundaries are shown below on cross-sectional OCT. Macular vascular density was separately calculated in three regions. Fovea region: $1 \mathrm{~mm}$ diameter circle centered on the avascular zone. Parafovea region: An annular area centered on the avascular zone with an inner diameter of $1 \mathrm{~mm}$ and an outer diameter of $3 \mathrm{~mm}$. Perifovea region: An annular area from the outside of parafoveal region to $6 \mathrm{~mm}$ diameter circle. 
SCP

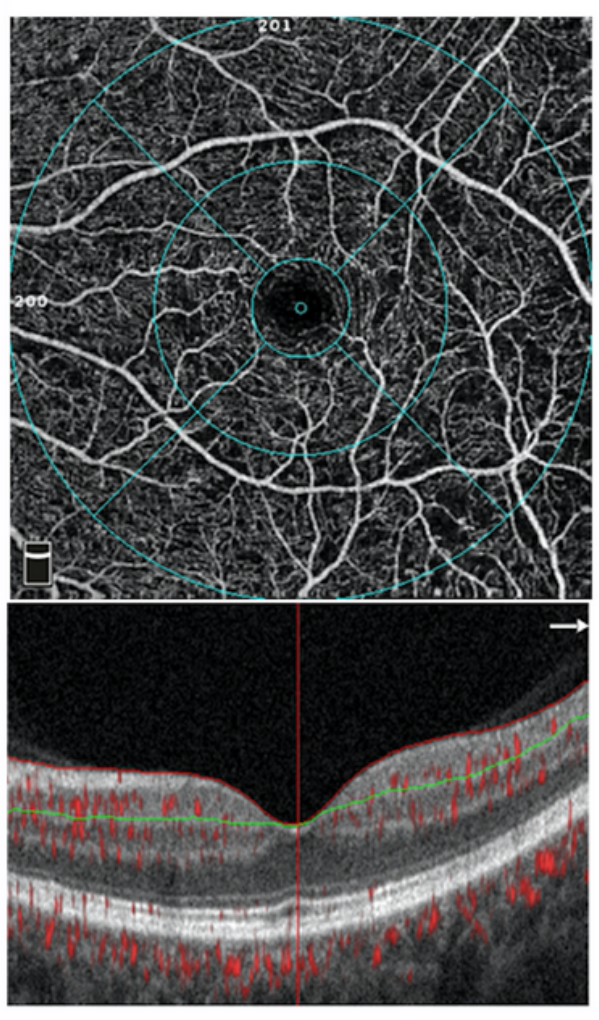

DCP
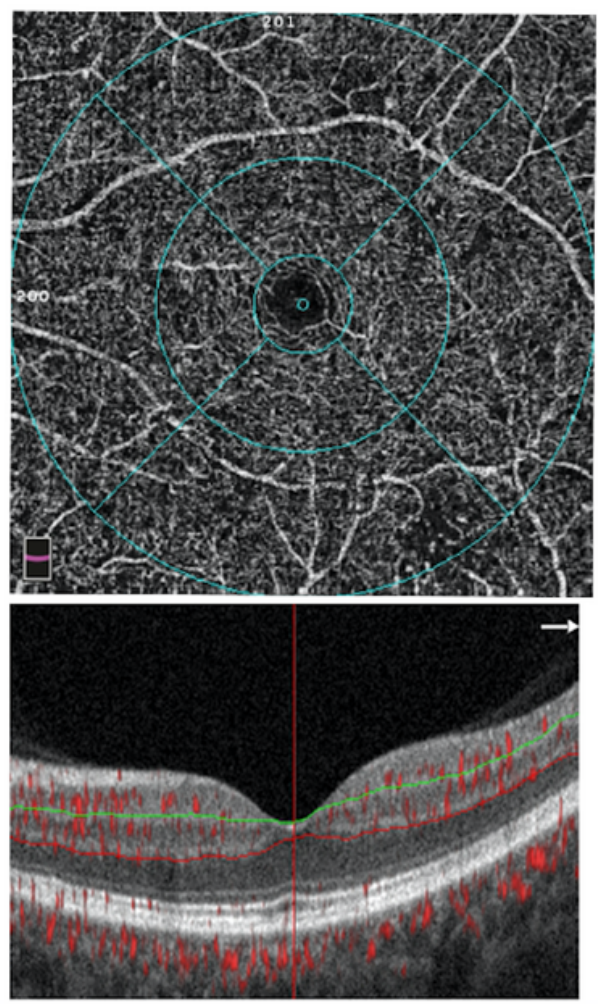

FAZ
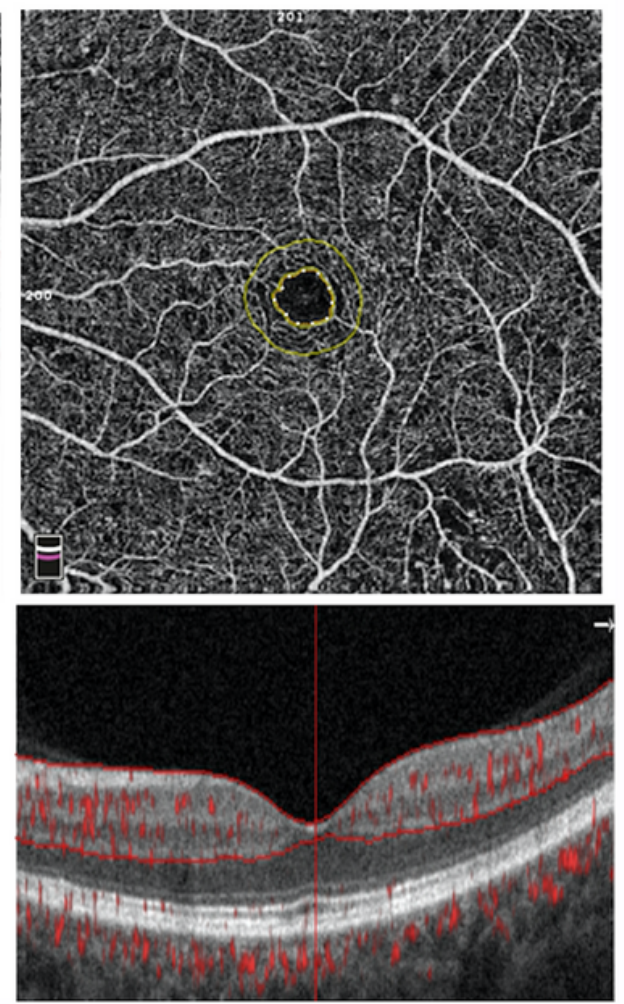

\section{Figure 1}

The segmentation of retinaEn face OCTA image of SCP(left),DCP(middle) and FAZ (right) of a S-NPDR eye. Segmentation boundaries are shown below on cross-sectional OCT. Macular vascular density was separately calculated in three regions. Fovea region: $1 \mathrm{~mm}$ diameter circle centered on the avascular zone. Parafovea region: An annular area centered on the avascular zone with an inner diameter of $1 \mathrm{~mm}$ and an outer diameter of $3 \mathrm{~mm}$. Perifovea region: An annular area from the outside of parafoveal region to $6 \mathrm{~mm}$ diameter circle. 
SCP

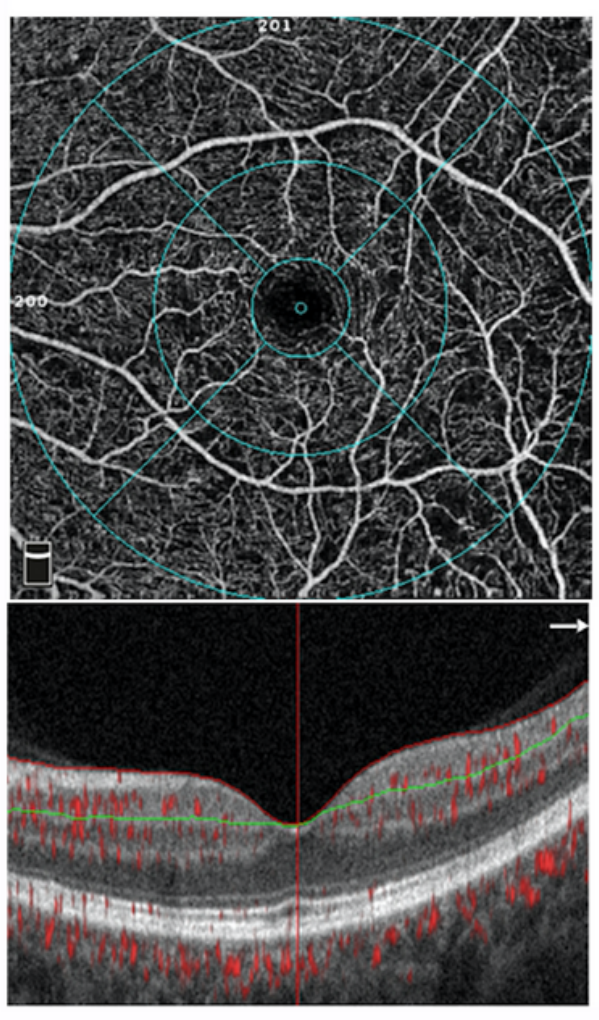

DCP
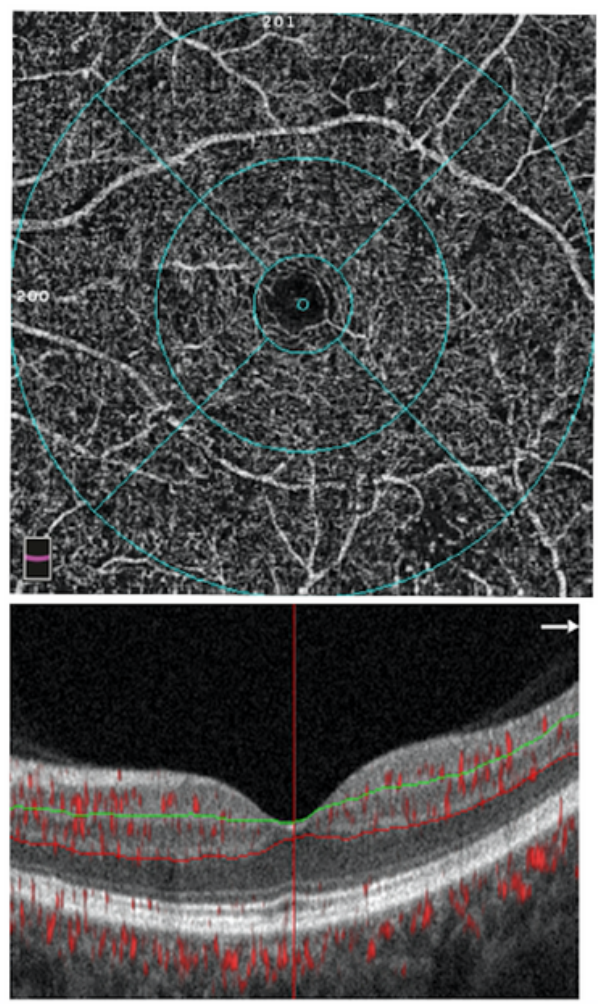

FAZ
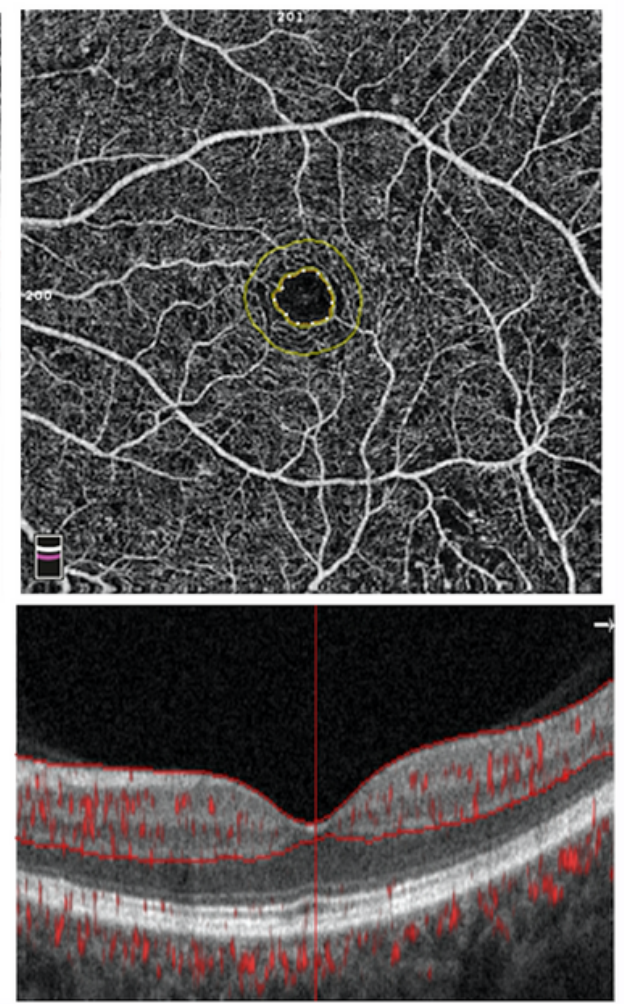

\section{Figure 1}

The segmentation of retinaEn face OCTA image of SCP(left),DCP(middle) and FAZ (right) of a S-NPDR eye. Segmentation boundaries are shown below on cross-sectional OCT. Macular vascular density was separately calculated in three regions. Fovea region: $1 \mathrm{~mm}$ diameter circle centered on the avascular zone. Parafovea region: An annular area centered on the avascular zone with an inner diameter of $1 \mathrm{~mm}$ and an outer diameter of $3 \mathrm{~mm}$. Perifovea region: An annular area from the outside of parafoveal region to $6 \mathrm{~mm}$ diameter circle. 
SCP
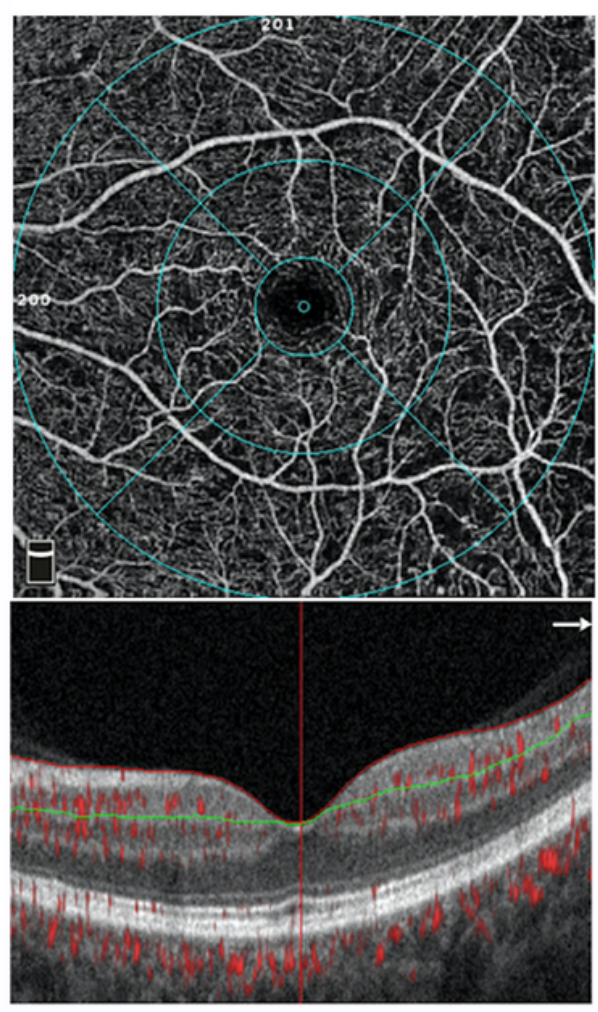

DCP
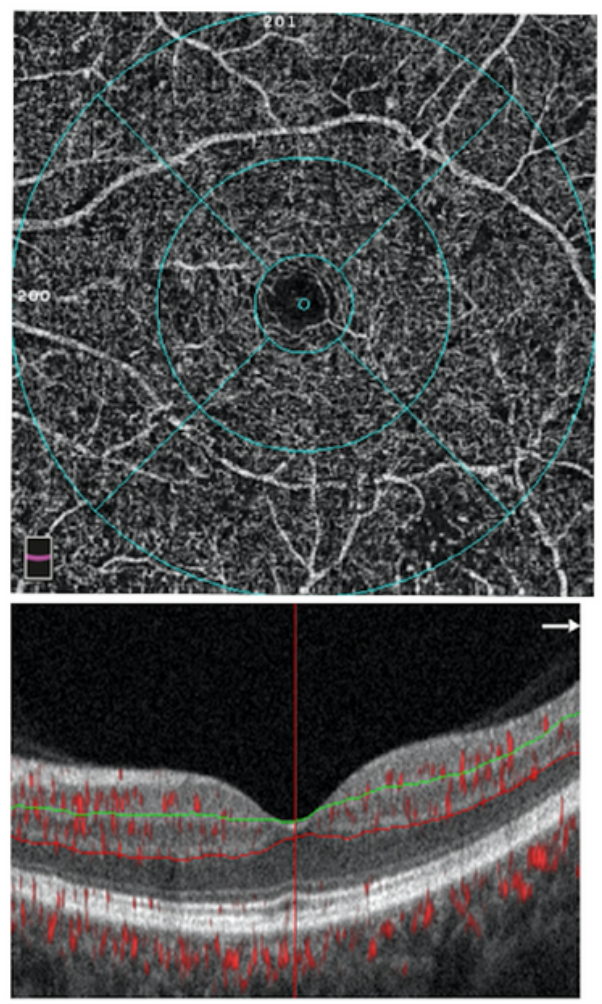

FAZ
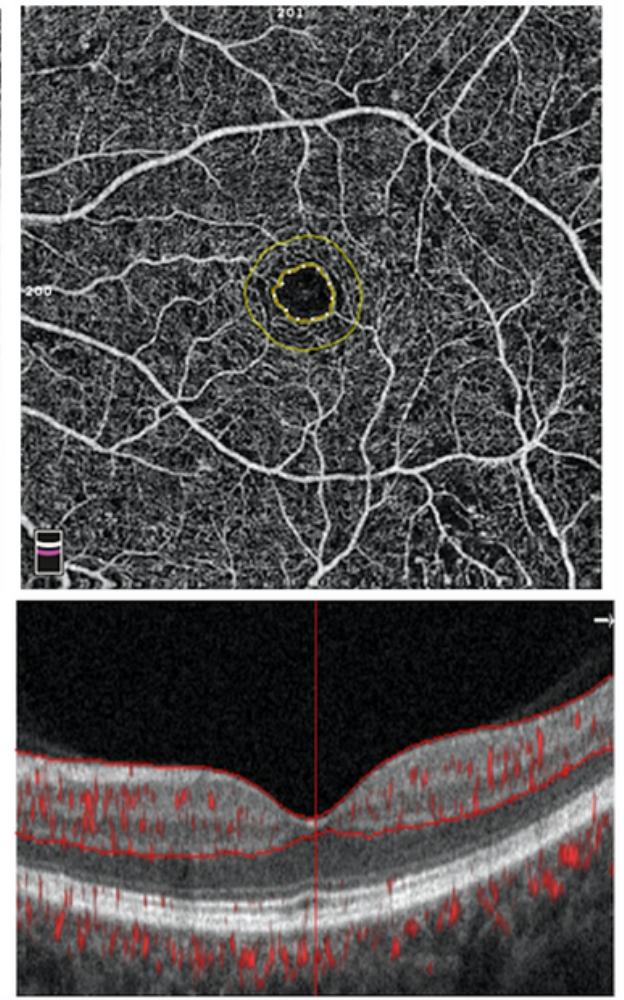

\section{Figure 1}

The segmentation of retinaEn face OCTA image of SCP(left),DCP(middle) and FAZ (right) of a S-NPDR eye. Segmentation boundaries are shown below on cross-sectional OCT. Macular vascular density was separately calculated in three regions. Fovea region: $1 \mathrm{~mm}$ diameter circle centered on the avascular zone. Parafovea region: An annular area centered on the avascular zone with an inner diameter of $1 \mathrm{~mm}$ and an outer diameter of $3 \mathrm{~mm}$. Perifovea region: An annular area from the outside of parafoveal region to $6 \mathrm{~mm}$ diameter circle. 

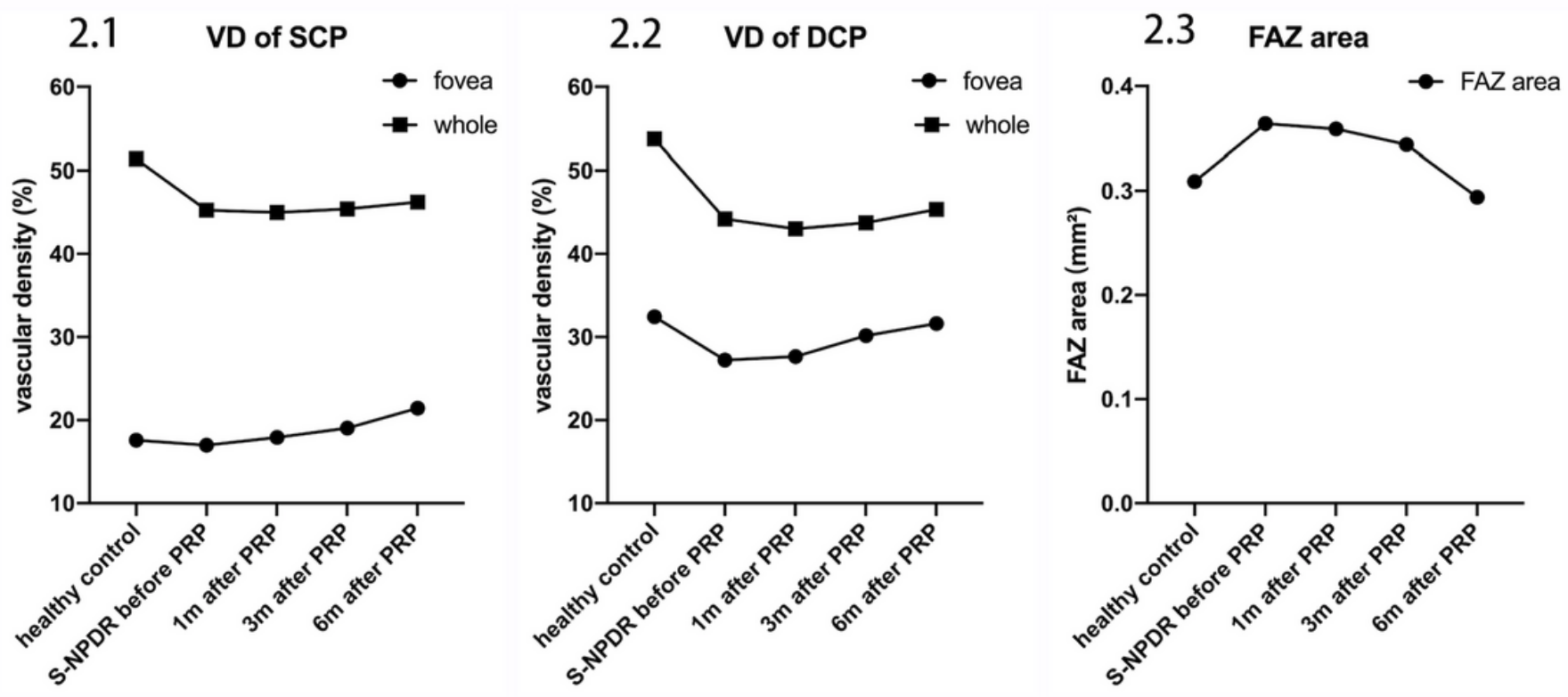

Figure 2

The trend of VD and FAZ area in normal eyes, S-NPDR eyes before PRP and S-NPDR eyes after PRP
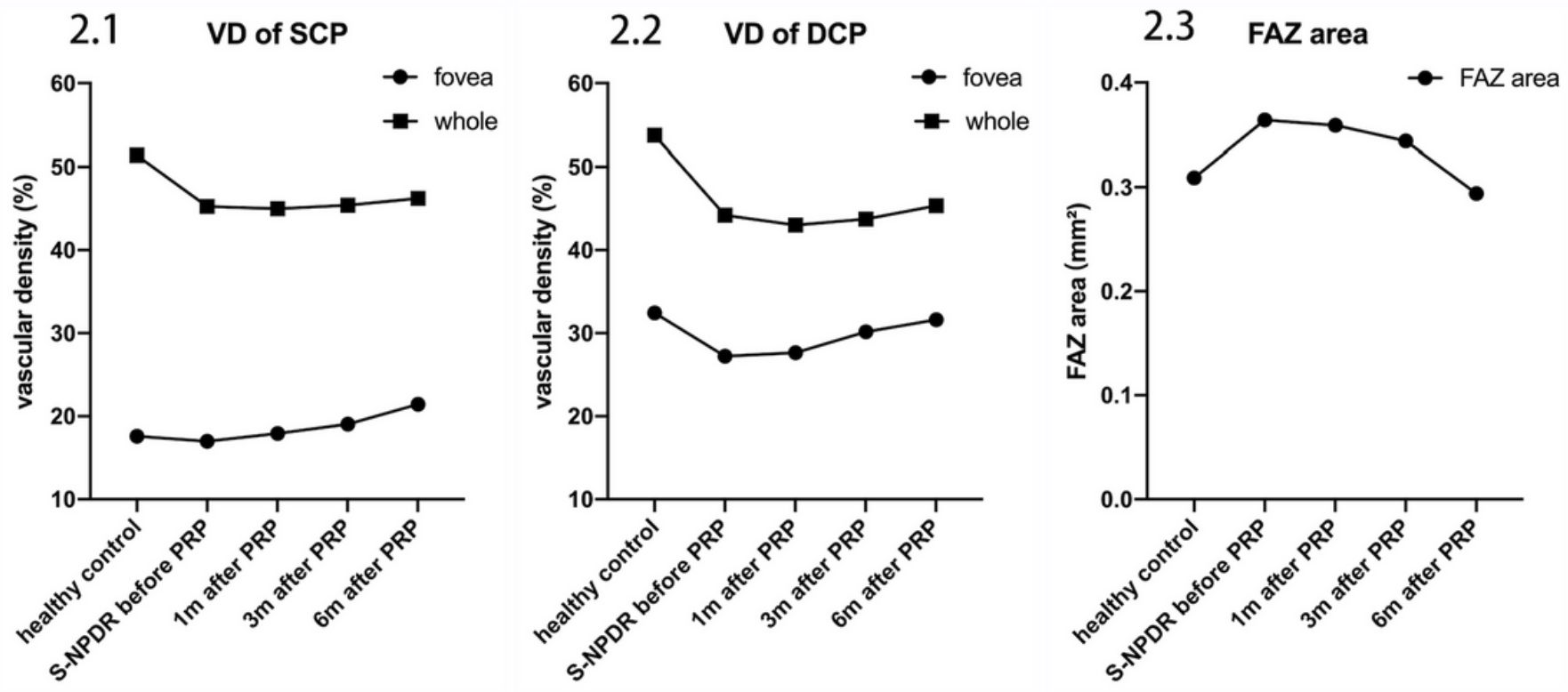

Figure 2

The trend of VD and FAZ area in normal eyes, S-NPDR eyes before PRP and S-NPDR eyes after PRP 

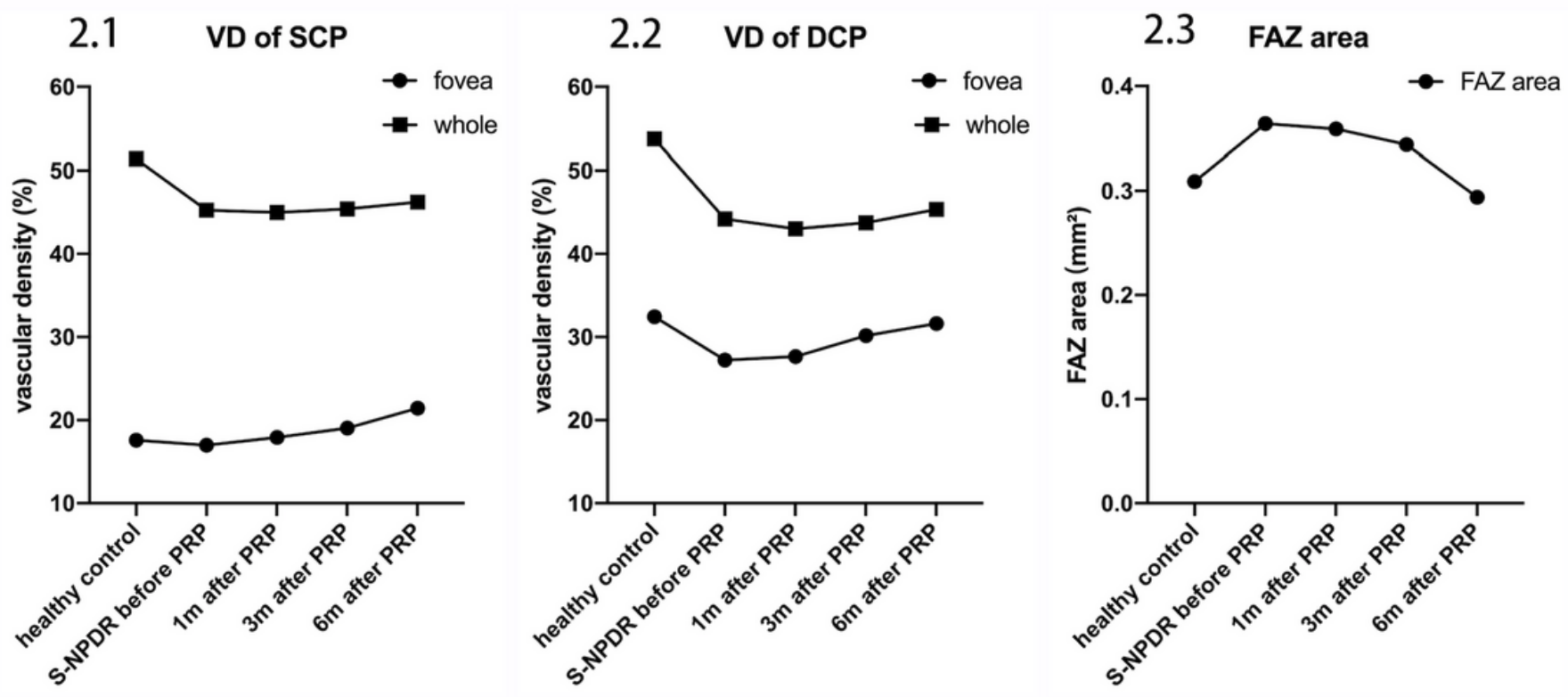

Figure 2

The trend of VD and FAZ area in normal eyes, S-NPDR eyes before PRP and S-NPDR eyes after PRP
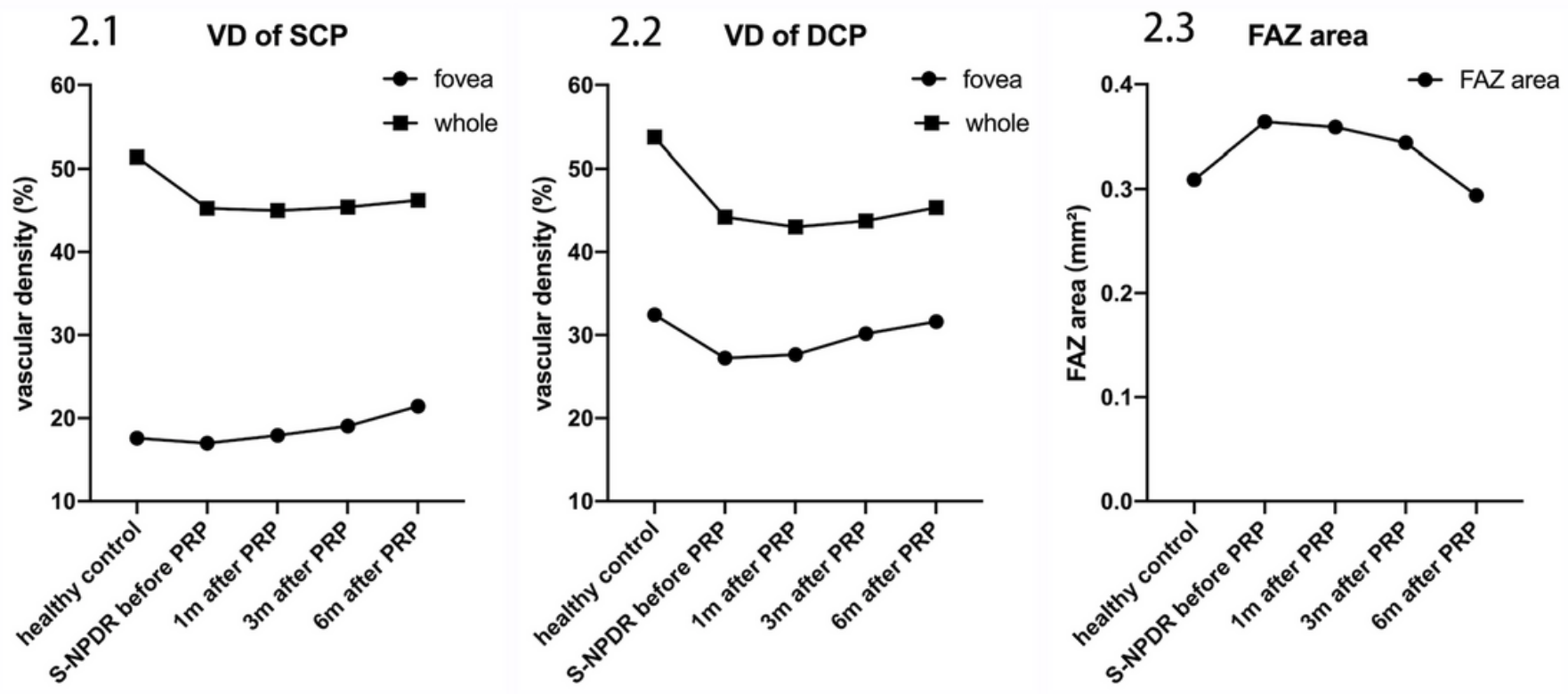

Figure 2

The trend of VD and FAZ area in normal eyes, S-NPDR eyes before PRP and S-NPDR eyes after PRP 